\title{
La Psicología Ambiental en el Siglo 21: El Desafío del Desarrollo Sustentable
}

\section{Environmental Psychology for The $21^{\text {st }}$ Century: The Challenge of Sustainable Development}

\author{
Gabriel Moser ${ }^{1}$
}

\begin{abstract}
Resumen
Con la llegada del Nuevo milenio, la psicología ambiental necesita tomar en cuenta dos variables que fueron, hasta hoy, consideradas de manera inadecuada: las diferencias interculturales y la dimensión temporal de las relaciones individuo - medio ambiente. El factor cultural no puede ser ignorado en las sociedades occidentales que son crecientemente interculturales, y nuestra relación tanto con el ambiente construido como con el ambiente natural necesita ser analizado en términos de temporalidad.

Este trabajo analiza el impacto de los factores culturales y de la perspectiva temporal en los cuatros niveles de análisis: el micro-ambiente, el ambiente próximo, el ambiente urbano y el ambiente global. Interesarse en esos cuatro niveles y sus interelaciones es particularmente importante ya que tenemos que cumplir con las exigencias del desarrollo sustentable con respecto al bienestar y la calidad de vida del individuo que son dependientes de necesidades culturalmente específicas.
\end{abstract}

Palabras claves: Psicología ambiental, desarrollo sustentable, dimensión temporal, diferencias interculturales, calidad de vida.

\begin{abstract}
With the millennium upon us, environmental psychology needs to take account of two variables which have, until now, been inadequately considered: intercultural differences and the temporal dimension of people - environment relations. The cultural factor cannot be ignored in western societies which are increasingly intercultural, and our relation to the built as well as the natural environment needs to be analysed in terms of temporality.

This paper analyses the impact of intercultural factors and the time perspective on four levels of analysis the micro-environment, the proximate environment, urban
\end{abstract}

1 CNRS Laboratoire de Psychologie Environnementale, UMR 8069 Université René Descartes-Paris 5. E-mail: gabriel.moser@univ-paris5.fr 
environment and the global environment. To look at these four levels and at their interrelations is particularly important when we have to meet the sustainable development exigencies towards the individual's well-being and quality of life, which are dependent on cultural specific needs.

Key words: environmental psychology, sustainable development, temporal dimension, intercultural differences, quality of life

En este inicio del siglo 21 dos referencias mayores son susceptibles de marcar profundamente el desarrollo de nuestras sociedades: la globalización y el desarrollo sustentable, conceptos claves de este nuevo siglo que tienen una repercusión importante sobre el modo de vida en general.

La aparición de preocupaciones concernientes al desarrollo sustentable da un nuevo impulso al desarrollo de la psicología ambiental. Las preocupaciones hasta entonces centradas en torno al hábitat y a la ciudad se extienden paulatinamente por una parte hacia el modo de vida y por la otra hacia los comportamientos ecológicos. Mas específicamente, el "Informe Brundtland" (Brundtland, 1997), que define el desarrollo sustentable como "un desarrollo susceptible de satisfacer las necesidades de la generación actual sin comprometer las posibilidades para las generaciones futuras de satisfacer las suyas", abre la vía a las preocupaciones vinculadas al modo de vida. La referencia a las necesidades permite en efecto incluir no solamente la necesidad de un desarrollo económico armonioso y respetuoso del ambiente, sino igualmente preocuparse del bienestar del individuo. Esta preocupación interpela particularmente a nuestra disciplina, en la medida en que los modos de relación con el ambiente constituyen un aspecto importante que contribuye al bienestar del individuo. En este marco de cosas, la psicología ambiental a través de la aportación de conocimientos pertinentes, resulta un medio fundamental para analizar, explicar, esclarecer y comprender las condiciones de bienestar, haciendo posible de esta manera la toma de decisiones en materia de ambiente.
La globalización de la economía y su corolario, la mundialización de intercambios y de medios de comunicación implica una presión a la uniformación cultural y de los modos de vida. La puesta en escena progresiva de la globalización ha significado estrictamente un miedo a la uniformización de los valores y al anonimato. En este sentido, la globalización es considerada por muchos de nuestros conciudadanos como amenazante para su identidad, y provoca una reivindicación identitaria. La globalización y los temores que la acompañan son así generadores de reivindicaciones identitarias con frecuencia ancladas territorialmente. Se trata innegablemente de una búsqueda de identidad espacialmente justificada. Esta condición representa el segundo mayor desafío a la psicología ambiental de este cambio de siglo. La investidura local, la apropiación y la identidad ambiental, son efectivamente conceptos utilizados tradicionalmente por nuestra disciplina, pero su inscripción dentro del desarrollo sustentable y la globalización les otorgan una nueva dimensión.

La unidad de análisis de la psicología ambiental es la relación individuo ambiente. Por su naturaleza misma, esta relación particular se pone en evidencia solamente sobre el terreno.

De hecho, la disciplina funciona según una lógica inductiva, las teorías se generan a partir de constataciones o de hechos que se ponen a prueba sobre el terreno. Asimismo las referencias a las exigencias de Lewin (1936), concernientes a la relación integral entre teoría y aplicación son citadas frecuentemente como modelo de funcionamiento de 
la psicología ambiental. La orientación de la psicología ambiental es tanto teórica como dirigida a la resolución de problemas. Es decir, se ubica como una disciplina de la psicología que elabora sus propias aproximaciones teóricas y como una aproximación en la cual la investigación y la aplicación están íntimamente ligadas. Si bien la psicología ambiental tiene fuertes afinidades con la mayor parte de otras ramas de la psicología, integra una dimensión importante a la psicología volviendo inteligibles las diferencias en los comportamientos o percepciones debidas a las variables contextuales, diferencias que no pueden ser explicadas más que en referencia a las contingencias ambientales (Moser \& Uzzell, 2002). Las exigencias del desarrollo sustentable y la globalización se deben orientar hacia la reflexión teórica tomando en cuenta de manera expresa la relatividad cultural de las necesidades, las dimensiones espaciales del bienestar y de la identidad, y por último, las cuestiones de la relación con el ambiente global.

\section{Las necesidades en materia de ambiente,} dentro de una perspectiva de globalización, formulan la cuestión de su universalidad. Esas necesidades ¿son las mismas en todas partes? Las investigaciones en psicología ambiental por ejemplo, nos han mostrado por una parte que la necesidad de espacio es diferente de una cultura a la otra y por otra que es diferente en función del ciclo de vida. Las investigaciones en psicología ambiental de carácter longitudinal orientadas a las comparaciones interculturales deben poder responder a estas cuestiones.

El bienestar no puede ser independiente de un anclaje territorial y de los procesos de identidad. Es así como esto reviste una importancia capital en el seno de las grandes metrópolis que se desarrollaron en el curso del siglo pasado. La psicología ambiental ha puesto en evidencia las condiciones de vida dentro de estos grandes centros urbanos: anonimato, inseguridad, indiferencia hacia los demás y exposición a una variedad de stress. De esta manera la ciudad no es más que una identidad indiferenciada, se debe considerar como compuesta por una gran cantidad de ambientes diferentes que se superponen y que son cada uno apropiables o apropiados para los respectivos habitantes, parece ser que este último aspecto se ha dejado un tanto del lado. En otros términos, ¿como los fenómenos de apropiación que permiten a la mayoría de los ciudadanos experimentar el sentimiento de " sentirse en su casa», se articulan con una manera de investir positivamente la ciudad? ¿Cuales son las condiciones individuales y ambientales de un sentimiento de "sentirse en su casa», y de una expr esión identitaria generadora de bienestar?

Las investigaciones concernientes a la adopción de los comportamientos compatibles con la preservación del ambiente estos últimos años se han beneficiado de un nuevo impulso derivado de los análisis en términos de ideologías (Poortinga, Steg, \& Vlek, 2002; Sjöber g, 1998 ; Steg \& Sievers, 2000 ; Thompson, Ellis \& Wildavsky, 1990) y a través de su referencia a las representaciones sociales (Moser, et al., 2002). ¿Bajo que condiciones los individuos son susceptibles de actuar en favor de una economía de recursos naturales? ¿Como las diferencias interculturales, especialmente en lo que concierne a los valores, son compatibles con los comportamientos pro-ambientales benéficos para las generaciones futuras?

\section{El objetivo de la psicología ambiental es} el de identificar los procesos que regulan y median la relación del individuo con el medio ambiente, poniendo en evidencia por una parte las percepciones, actitudes, evaluaciones y representaciones ambientales y por la otra los comportamientos y conductas ambientales que los acompañan. La psicología ambiental se interesa tanto a los efectos de las condiciones ambientales sobre los comportamientos y conductas como a la manera en la 
cual el individuo percibe o actúa sobre el ambiente. Si bien los análisis pueden tomar como punto de partida tanto al individuo como ciertos aspectos del ambiente ya sea físicos (ruido, contaminación, acondicionamiento del cuadro urbano) o sociales (densidad, heterogeneidad de la población), estos análisis desembocan con frecuencia mas allá del esclarecimiento de las incidencias específicas de los aspectos mencionados hacia una explicación interrelacional y sistemática. Lo anterior, en la medida en que los factores físicos son intrincablemente vinculados a los efectos que producen sobre la percepción y el comportamiento del individuo (Altman y Rogoff, 1987).

Tanto el desarrollo sustentable como la globalización de intercambios movilizan el conjunto de temáticas desarrolladas tradicionalmente por la psicología ambiental. Efectivamente, el hábitat, los modos de vida urbanos, las necesidades en materia de ambiente, la implicación local y la investigación sobre identidad así como las actitudes y los comportamientos en favor del ambiente son temáticas que han preocupado a la psicología ambiental desde sus inicios en los años 70 . Pero actualmente se debe reintegrar el conjunto de estas temáticas dentro de una pers- pectiva intercultural y dentro de una dinámica temporal (cf. Figura 1). Esta integración conduce a ampliar la definición de la psicología ambiental considerándola como el estudio de las interrelaciones entre el individuo y su ambiente físico y social, dentro de sus dimensiones espaciales y temporales.

Si bien se adivina su presencia, la dimensión temporal en su relación con el ambiente físico y social ha estado profundamente olvidada en la psicología ambiental. Los enfoques en términos de transacción se inscriben en este sentido dentro de una transformación mutua a largo plazo. El sistema de interacciones-transacciones entre el individuo y el ambiente integra tanto su experiencia como sus proyectos, sus representaciones y sus acciones, y por lo tanto se inscriben dentro de la temporalidad. La capacidad del individuo para proyectarse dentro del futuro adquiere toda su importancia a partir del análisis de las condiciones de adopción de comportamientos ecológicos. Las referencias al tiempo están presentes de manera recurrente en psicología ambiental. Los autores como Altman et Rogoff (1987), Proshansky (1987), así como recientemente Werner et al., (1992) refieren explícitamente lo anterior.

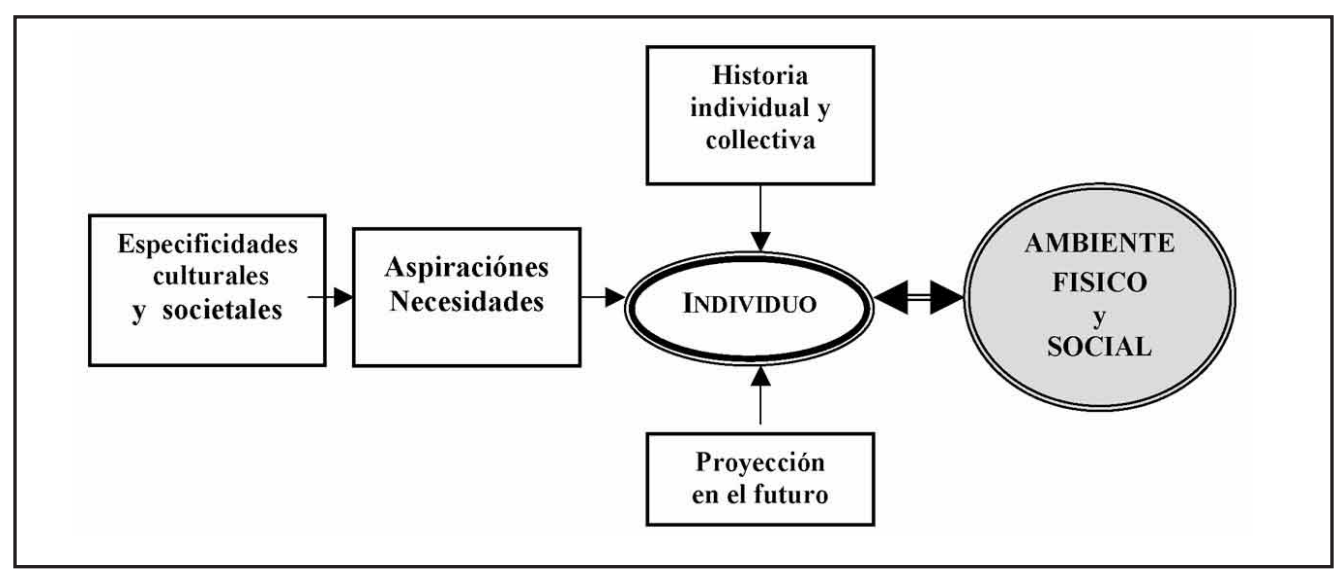

Fig. 1. La Relación del Individuo con el Ambiente en función de su Posición Temporal y Cultural 
A partir de su objeto la psicología ambiental ha sido y es ante todo una psicología del espacio, en la medida en que analiza las percepciones, las actitudes y los comportamientos del individuo en relación explícita con el contexto físico y social dentro del cual evoluciona. La psicología ambiental funciona así dentro de diversos niveles de referencia espacial. Es de esta manera como podemos distinguir cuatro niveles, (1) el micro-ambiente (el espacio privado: la habitación, el espacio privado en el lugar de trabajo); (2) los ambientes de proximidad (los espacios compartidos, espacios semi-públicos, habitación colectiva, barrio, el lugar de trabajo, parques, espacios verdes); (3) los ambientes públicos (ciudades, pueblos, campo, paisaje), etc., y (4) el ambiente global (el ambiente en su totalidad: englobando el ambiente construido y el ambiente natural; los r ecursos naturales).

Este funcionamiento implica no solamente un aspecto físico, pero también un aspecto social implicando a cada nivel mas gente y relaciones mas distantes (cf. Figura 2).

\begin{tabular}{|c|c|c|c|}
\hline & ambiente físico & ambiente social & $\begin{array}{l}\text { tipo de espacio } \\
y \text { de control }\end{array}$ \\
\hline Nivel I & $\begin{array}{c}\text { micro-ambiente } \\
\text { hábitat } \\
\text { espacio de trabajo }\end{array}$ & $\begin{array}{l}\text { nivel individual } \\
\text { familia }\end{array}$ & $\begin{array}{l}\text { espacios privados } \\
\text { control extendido }\end{array}$ \\
\hline Nivel II & $\begin{array}{l}\text { ambientes próximos } \\
\text { vecinancia, espacios } \\
\text { abiertos al publico }\end{array}$ & $\begin{array}{c}\text { nivel interindividual } \\
\text { comunidad } \\
\text { usadores, clientes }\end{array}$ & $\begin{array}{l}\text { espacios semi-públicos } \\
\text { control compartido }\end{array}$ \\
\hline Nivel III & $\begin{array}{l}\text { ambientes públicos } \\
\text { ciudades, pueblos }\end{array}$ & $\begin{array}{c}\text { habitantes } \\
\text { conglomerado de } \\
\text { individuos }\end{array}$ & $\begin{array}{l}\text { espacios públicos } \\
\text { control mediatizado }\end{array}$ \\
\hline Nivel IV & ambiente global & $\begin{array}{c}\text { sociedad } \\
\text { populación }\end{array}$ & $\begin{array}{c}\text { país, nación } \\
\text { planeta } \\
\text { ausencia de control }\end{array}$ \\
\hline
\end{tabular}

Fig. 2. Aspectos Físicos y Sociales de los Diferentes Niveles del Ambiente

En referencia al nivel de análisis, es posible derivar un aspecto fundamental de la relación individuo-ambiente: las posibilidades de control y dominio del ambiente correspondiente (Fisher, 1984; 1986). Esta posibilidad permite al individuo dominar dentro de ciertos márgenes los diferentes aspectos del ambiente. La percepción de una pérdida de control,- por ejemplo, debido a una situación de malestar o nociva,- produce stress (Averill, 1973; Moser, 1992) y tiene consecuencias negativas no solamente en el comportamiento, sino también en el bienestar y la salud del individuo (Barnes, 1981).

De manera evidente, la dimensión cultural y la dimensión temporal intervienen en diversos grados en cada uno de los niveles arriba mencionados.

Las posibilidades de control individual y directo son características en el caso del microambiente, la apropiación no es compartida y en consecuencia el control es absoluto. 
En los espacios compartidos, espacios semi-públicos, como las habitaciones colectivas, el barrio o el lugar de trabajo, las posibilidades de control y dominio dejan de ser individuales y pasan a ser compartidas con otras personas. A este nivel la participación se hace posible y puede surgir un sentido de pertenencia, pero el control es siempre colectivo, mediado por otros y basado en un cierto consenso.

A nivel público, el control está más allá del alcance del individuo. En este caso el control sólo puede ser un control colectivo o social, mediado, otorgado y determinado necesariamente por el conjunto de personas.

Al nivel global el control va más allá del poder del individuo. El sentimiento de responsabilidad relativo a los problemas ambientales disminuye a través de esta distancia debido a un sentimiento de impotencia en el nivel global (Uzzell, 2000). Esto deriva en que la manera en que el ambiente nos afecta es casi exclusivamente analizado en términos de estrategias de confrontación y no en términos de dominio. Ya que las posibilidades de control individual, compartido o mediado, son inexistentes.

Ciertas investigaciones sobre las condiciones de un bienestar individual (Moser, et al., 2002) muestran que el bienestar esta correlacionado con una apreciación positiva de la población en relación a su entorno de vida, esto se manifiesta a través de los encuentros frecuentes con otros habitantes del barrio y la relación con ellos que van mas allá de una simple cortesía, así como en un sentimiento de seguridad. Tales condiciones permiten la expresión de una identidad residencial urbana y un sentimiento de pertenencia a una comunidad urbana. En otros términos, el bienestar individual depende de la posibilidad de una apropiación ambiental.
El bienestar individual, a través de una apropiación ambiental, permite además una predisposición positiva hacia el ambiente que constituye una condición importante para los comportamientos ecológicos. En efecto, el individuo está dispuesto a adoptar comportamientos pro-ambientales si logra identificar el problema, si el contexto social es favorable, es decir si el individuo experimenta un sentimiento de pertenecer a una comunidad con la cual se siente solidario y si posee un sentimiento de poder ejercer algún grado de control.

En conclusión: La historia de la psicología ambiental estuvo en sus inicios fuertemente influenciada por las cuestiones que destacan los arquitectos, los urbanistas $\mathrm{u}$ otros profesionales del acondicionamiento del cuadro urbano. La psicología ambiental a través de la ingeniería socio-ambiental, se encuentra en condiciones de contribuir significativamente a la solución de los grandes problemas de la sociedad planteados por las exigencias del desarrollo sustentable (Moser, 2002). En efecto el bienestar del individuo que se establece como prioridad dentro del informe Bruntland sobre el desarrollo esperado para los próximos años, interpela directamente a la psicología ambiental en este inicio del siglo 21. De esta manera, es indispensable tomar en cuenta la dimensión temporal y la dimensión cultural en cuanto a la calidad de vida. El bienestar depende de la satisfacción de necesidades culturalmente determinadas. El apego ambiental y su apropiación constituyen la base de la identidad ambiental, son procesos progresivos que son esenciales para el bienestar individual y para el surgimiento de comportamientos favorables al desarrollo sustentable. La relación del individuo con el ambiente que va desde el hábitat, pasando por la ciudad hasta el ambiente global, depende del nivel de control, es decir de la posibilidad de dominio que el individuo puede ejercer. 


\section{Referencias Bibliográficas}

Altman, I. \& Rogoff, B. (1987). World-views in psychology: trait, interactional, organismic and transactional perspectives, in: D. Stokols and I. Altman (Eds.) Handbook of Environmental Psychology. New York: Wiley, Vol. 1 pp. 7-40.

Averill J.R. (1973) - Personal control over aversive stimuli and its relationship to stress. Psychological Bulletin, 80, 286-303.

BARNES, R.D. (1981). Perceived freedom and control in the built environment. In J.H. Harvey (Ed.) Cognition, social behavior and the environment. Hillsdale N.J.: Erlbaum.

BRuntland, G.H., (1987). Our Common Future: Report of the World Commission on Environment and Development. Oxford: Oxford University Press.

FISHER, S. (1984) - Stress and the Perception of Control. London: Lawrence Erlbaum Associates Ltd.

FISHER, S. (1986) - Stress and Strategy. London: Lawrence Erlbaum Associates Ltd.

Moser, G. (1992). Les stress urbains. Paris: A. Calin, 193p.

Moser, G. (2002). People, places and sustainability: an agenda for the future. in: G. Moser, E. Pol, Y. Bernard, M. Bonnes, J. Corraliza \& V. Giuliani (Eds.) People, Places E Sustainability. Göttingen, Germany:Hogr efe \& Huber.
Moser, G. \& Uzzell, D. (2002). Environmental psychology. in: Millon, T., \& Lerner, M.J. (Eds.), Comprehensive Handbook of Psychology, Volume 5: Personality and Social Psychology, New York: John Wiley \& Sons.

Moser, G., Ratiu, E. \& Fleury-Bahi, G. (2002). Appropriation and interpersonal relationships: from dwelling to city through the neighbourhood. Environment $\mathcal{E}$ Behavior, (Special issue) 34(1), 122-136.

Poortinga, W., Steg, L. \& Vlek, C. (2002) Myths of nature and environmental management strategies. A field study on energy savings in traffic and transport. in: E. Pol, Y. Bernard, M. Bonnes, J. Corraliza \& V. Giuliani (Eds.) People, Places $\mathcal{E}$ Sustainability. Göttingen, Germany:Hogr efe \& Huber.

Proshansky, H. M., Fabian, A. K. \& Kaminoff, R. (1983) 'Place identity: Physical world socialisation of the self', Journal of Environmental Psychology, 3, 57-83.

Thompson, M., Ellis, R., \& Wildavsky, A. (1990). Cultural Theory. Boulder, CO: Westview Press.

Uzzell, D.L. (2000). The Psycho-Spatial Dimension to Global Environmental Problems, Journal of Environmental Psychology, 20, 3, 307-318.

Werner C.M., Altman, I. \& Brown B.B. (1992). A transactional approach to interpersonal relations: physical environment, social context and temporal qualities. Journal of Social and Personal Relationships, 9, 297-323. 